\title{
Glucosinolate and isothiocyanate intakes are inversely associated with breast cancer risk: a case-control study in China
}

\author{
Nai-Qi Zhang ${ }^{1,2}$, Suzanne C. Ho ${ }^{3}$, Xiong-Fei Mo ${ }^{4}$, Fang-Yu Lin ${ }^{5}$, Wu-Qing Huang ${ }^{1}$, Hong Luo ${ }^{1}$, \\ Jing Huang ${ }^{1}$ and Cai-Xia Zhang ${ }^{1,2 *}$ \\ ${ }^{1}$ Department of Medical Statistics and Epidemiology, School of Public Health, Sun Yat-sen University, Guangzhou 510080, \\ People's Republic of China \\ ${ }^{2}$ Guangdong Provincial Key Laboratory of Food, Nutrition and Health, School of Public Health, Sun Yat-sen University, \\ Guangzhou 510080, People's Republic of China \\ ${ }^{3}$ Division of Epidemiology, The Jockey Club School of Public Health and Primary Care, The Chinese University of Hong Kong, \\ Hong Kong SAR, People's Republic of China \\ ${ }^{4}$ Department of Thyroid and Breast Surgery, the First Affiliated Hospital of Sun Yat-sen University, Guangzhou 510080, \\ People's Republic of China \\ ${ }^{5}$ Nursing Department, the First Affiliated Hospital of Sun Yat-sen University, Guangzhou 510080, People's Republic of China \\ (Submitted 18 November 2017 - Final revision received 29 January 2018 - Accepted 15 February 2018)
}

\section{Abstract}

Although previous studies have investigated the association of cruciferous vegetable consumption with breast cancer risk, few studies focused on the association between bioactive components in cruciferous vegetables, glucosinolates (GSL) and isothiocyanates (ITC), and breast cancer risk. This study aimed to examine the association between consumption of cruciferous vegetables and breast cancer risk according to GSL and ITC contents in a Chinese population. A total of 1485 cases and 1506 controls were recruited into this case-control study from June 2007 to March 2017. Consumption of cruciferous vegetables was assessed using a validated FFQ. Dietary GSL and ITC were computed by using two food composition databases linking GSL and ITC contents in cruciferous vegetables with responses to the FFQ. The OR and 95\% CI were assessed by unconditional logistic regression after adjusting for the potential confounders. Significant inverse associations were found between consumption of cruciferous vegetables, GSL and ITC and breast cancer risk. The adjusted OR comparing the highest with the lowest quartile were 0.51 (95\% CI 0.41, 0.63) for cruciferous vegetables, 0.54 (95\% CI 0.44, 0.67) for GSL and 0.62 (95\% CI 0.50, 0.76) for ITC, respectively. These inverse associations were also observed in both premenopausal and postmenopausal women. Subgroup analysis by hormone receptor status found inverse associations between cruciferous vegetables, GSL and ITC and both hormone-receptor-positive or hormone-receptor-negative breast cancer. This study indicated that consumption of cruciferous vegetables, GSL and ITC was inversely associated with breast cancer risk among Chinese women.

Key words: Cruciferous vegetables: Glucosinolates: Isothiocyanates: Breast cancer: Case-control studies

Breast cancer is the most common cancer in women, and ranks as the leading cause of cancer-related death among women worldwide. In all, 1.7 million breast cancer cases consisted of onequarter of the emerging malignant tumours, and 521900 deaths occurred in $2012^{(1)}$. Although the incidence of breast cancer has been growing worldwide in the past years, the incidence in Asian countries is still lower compared with that in Western countries $^{(2)}$. Studies have shown evidence that the increasing breast cancer risk is associated with dietary factors, which could be a safe and cost-effective method for cancer prevention.

Cruciferous vegetables have been of great interest. Numerous meta-analyses have shown the beneficial effect of cruciferous vegetables on breast, lung, colorectal, gastric, pancreatic, renal, prostate and other cancers ${ }^{(3-11)}$. The anti-carcinogenic effect of cruciferous vegetables against various cancers may be owing to the high levels of glucosinolates (GSL) ${ }^{(12)}$, which can hydrolyse into indole-3-carbinol and isothiocyanates (ITC) by plant myrosinase and gastrointestinal microflora. Although GSL have no direct biological activity, its major hydrolysis products exert their anti-carcinogenic effects in vivo by multiple mechanisms, including inhibiting the activity of carcinogen-activating enzymes, expediting apoptosis and arresting cell cycle progression $^{(13)}$. ITC, in particular, is able to suppress the activation of carcinogen by cytochromes P450 (CYP) by down-regulating

Abbreviations: ER, oestrogen receptor; ER+, ER positive; ER-, ER negative; GSL, glucosinolate; ITC, isothiocyanate; PR, progesterone receptor; PR+, PR positive; PR-, PR negative.

* Corresponding author: Professor C.-X. Zhang, fax +86 20 87330446, email zhangcx3@mail.sysu.edu.cn 
the enzyme levels and directly inhibiting their catalytic activities. In addition, ITC can induce glutathione $S$-transferase (GST), one of the most crucial phase II enzymes, which can effectively detoxify electrophilic compounds generated by phase I enzymes and further destroy their ability to damage DNA ${ }^{(14)}$. As the main sources of these compounds are easily available in traditional Chinese diets, these agents are ideal for potential effective chemoprotection of humans against cancer.

One meta-analysis published in 2013 suggested that high consumption of cruciferous vegetables may reduce the risk of breast cancer ${ }^{(10)}$. Only one cohort study found the inverse association between consumption of GSL and prostate cancer $^{(15)}$. Two case-control studies observed that higher ITC intake was associated with lower lung cancer risk ${ }^{(16-17)}$. However, no epidemiological study has yet carried out systematic investigation between total cruciferous vegetables, estimated GSL and ITC content consumption and the risk of breast cancer. Thus, we performed a case-control study to evaluate the association between cruciferous vegetable intakes and breast cancer risk according to GSL and ITC content in a Chinese population. We hypothesised that high intake of cruciferous vegetables, GSL and ITC was inversely associated with breast cancer risk.

\section{Methods}

\section{Study population}

A two-stage hospital-based case-control study was conducted to recruit breast cancer cases and controls. Detailed study methods of the first-stage case-control study design have been reported elsewhere ${ }^{(18-19)}$. To be brief, the first stage was conducted from June 2007 to August 2008 and the second stage from September 2011 to March 2017. Eligible women aged 25-70 years, Guangdong natives or those who have lived in Guangdong province for at least 5 years with incident, primary, histologically confirmed breast cancer diagnosed no more than 3 months before the interview were recruited from three major hospitals in Guangzhou. Subjects were excluded if they could not understand or speak Mandarin or Cantonese or had a past history of cancers. Totally, 1614 eligible cases were identified, of which 1485 (92.0\%) were successfully interviewed.

Controls were admitted to the same hospitals as cases during the same time period, frequency-matched by age (5-year interval) with cases. Control subjects shared the same eligibility criteria with cases, except that they had no past history of any cancer. They were selected from the Departments of Ophthalmology, Plastic and Reconstructive Surgery, Vascular Surgery, Ear-Nose-Throat, and Orthopedics and Microsurgery. In total, 1506 (92.4\%) controls out of 1630 eligible controls participated in this study.

We assumed that people with a higher intake of cruciferous vegetables represented $25 \%$ of the general population, the estimated OR between the consumption of cruciferous vegetables and breast cancer risk was $0.49^{(19)}$, the type I error rate was $<0.05(\alpha=0.05)$, the power of test was $90 \%(\beta=0 \cdot 10)$ and the response rate was $90 \%$. On the basis of these assumptions, we require a sample size of 304 cases.
The present study was conducted according to the guidelines laid down in the Declaration of Helsinki. All procedures involving human subjects were approved by The Ethical Committee of School of Public Health, Sun Yat-sen University. Written informed consents were obtained from all participants.

\section{Data collection}

A face-to-face interview was performed by trained interviewers by using a structured questionnaire, which comprised dietary habits, socio-demographic factors, anthropometric factors, lifestyle behaviours, family history of cancer and reproductive information. In this study, regular smokers were defined as those smoking at least 1 cigarette/d for more than 6 consecutive months. Passive smokers were defined as non-smokers who reported being exposed to others' tobacco smoke at least $15 \mathrm{~min} / \mathrm{d}$ in the previous 5 years. Regular drinkers were defined as those drinking alcohol at least once per week in the past 5 years. Women were identified as postmenopausal if they had cessation of menstrual period for at least 12 months since the last menstrual cycle. BMI was calculated by dividing weight $(\mathrm{kg})$ by height squared $\left(\mathrm{m}^{2}\right)$. Data on current occupational activity were obtained on the basis of self-reported employment status and the level of physical activity done at work (non-working, sedentary, standing, manual, heavy manual). A metabolic equivalent (MET) value was assigned to each reported activity based on the Compendium ${ }^{(20-21)}$. MET-h per week (how many days per week $\times$ how many hours per day $\times$ MET for a specific activity) were calculated for the frequency (d/week) and typical duration ( $\mathrm{h} / \mathrm{d}$ ) of household activities (mopping, cooking and so on) and recreational activities (walking, jogging, climbing, running, playing table tennis and so on) during the past year. Relevant medical information, medical diagnosis, histological findings and oestrogen receptor (ER) and progesterone receptor (PR) status were extracted from the hospital medical records.

Cruciferous vegetable intakes were assessed using a validated ninety-one-item FFQ, which investigated the habitual diet of all the subjects during the previous year before diagnosis for cases or before the time of interview for controls. The validity and reliability of FFQ have been described previously ${ }^{(22)}$. Each participant was asked the frequency, on average, of each type of food they consumed over the previous year. Food photographs about different portion size of foods were used to help the participants to quantify the amount they consumed. Nutrient values intakes were estimated using the 2002 Chinese Food Composition Table ${ }^{(23)}$. A total of ten types of cruciferous vegetables common in Guangdong province were listed on the FFQ, including choi sum, kaichoi, kailan, broccoli, pakchoi, bokchoi, cabbage, mini Chinese cabbage, cauliflower and radish. On the basis of the reported intake information, the daily average intake of total cruciferous vegetable was calculated. Furthermore, dietary GSL was computed by linking food consumption data to a food composition database ${ }^{(24)}$, which summarised eighteen studies to form a database on total GSL contents in cruciferous vegetables. The estimated weekly intake of ITC was calculated by using previously published ITC concentrations for cruciferous vegetables grown in Singapore and cooked in boiling water ${ }^{(25)}$ by linkage of ITC contents in cruciferous vegetables with responses to the FFQ. 


\section{Statistical analysis}

Subjects were categorised into quartiles based on the distribution of cruciferous vegetables or nutrients among the controls. The lowest quartile served as the reference group in the analyses. Student's $t$ tests were used for continuous variables (such as age, BMI, household and recreational activities, age at menarche) and $\chi^{2}$ tests were used for categorical variables (such as income, educational level, smoking status) to test differences between different groups. Dietary cruciferous vegetables and nutrient intake were adjusted for total energy intake by using the residual method $^{(26)}$. OR and $95 \%$ CI summarising the association between cruciferous vegetables or nutrients and breast cancer risk were calculated by using unconditional logistic regression adjusting for age, education, income, BMI, regular smoking, passive smoking, regular drinking, household and recreational activities, occupational activity, age at menarche, first-degree relative with cancer and ever used an oral contraceptive. Confounding factors were selected by comparing baseline characteristics between the cases and controls. Tests for trend were performed by entering the categorical variables as continuous parameters in the models.

As menopause is the marker of cessation of ovarian function and related to endocrine activity, and late menopause is a known risk factor for breast cancer ${ }^{(27)}$, stratified analysis by menopausal status (premenopausal and postmenopausal) was conducted to assess the associations between cruciferous vegetable or nutrient intakes and breast cancer risk. Subgroup analysis by sex hormone receptor status (ER positive (ER+) or ER negative (ER-); PR positive (PR+) or PR negative (PR-)) was also conducted. All of the aforementioned statistical analyses were carried out using IBM SPSS Statistics, version 21.0.

\section{Results}

The socio-demographic and established breast cancer risk factors of the study subjects are shown in Table 1. Compared with controls, breast cancer cases were more likely to have less education and lower income. More cases reported having a first-degree relative with cancer, a history of using oral contraceptive, higher BMI and younger age at menarche. A higher proportion of cases tended to drink and smoke regularly and be exposed to second-hand smoke, engaged in fewer household and leisure activities and more occupational activities. These variables were considered potential confounders and adjusted for in the subsequent multivariable analyses. Age was also adjusted in the model as matching was on 5-year intervals. No significant differences were observed between cases and controls regarding age, marital status, occupation, age at first live birth, parity, menopausal status and breast-feeding history.

Among control subjects, mean intakes were 130.53 (SD 68.93$) \mathrm{g} / \mathrm{d}$ for energy-adjusted total cruciferous vegetables, $100.94(\mathrm{sD} 50 \cdot 80) \mathrm{mg} / \mathrm{d}$ for energy-adjusted GSL and $32 \cdot 10$ (SD $20 \cdot 79) \mu \mathrm{mol} / \mathrm{d}$ for energy-adjusted ITC. Compared with controls, cases tended to have lower intakes of total cruciferous vegetables, GSL and ITC (Table 1).

A strong inverse association was found between total cruciferous vegetable intake and breast cancer risk (Table 2). The OR for the highest quartile of intake in comparison with the lowest
Table 1. Socio-demographic characteristics and selected risk factors of breast cancer in the study population

(Mean values and standard deviations; numbers and percentages)

\begin{tabular}{|c|c|c|c|c|c|}
\hline & \multicolumn{2}{|c|}{ Cases ( $n$ 1485) } & \multicolumn{2}{|c|}{ Controls ( $n$ 1506) } & \multirow[b]{2}{*}{$P$} \\
\hline & Mean & SD & Mean & SD & \\
\hline Age (years) & 47.56 & 9.47 & 47.58 & 9.45 & 0.955 \\
\hline $\mathrm{BMl}\left(\mathrm{kg} / \mathrm{m}^{2}\right)$ & 22.98 & 3.38 & 22.59 & $3 \cdot 10$ & 0.001 \\
\hline $\begin{array}{l}\text { Household and } \\
\text { recreational activities } \\
\text { (MET-h/week) }\end{array}$ & $36 \cdot 41$ & 24.43 & 39.82 & $26 \cdot 28$ & $<0.001$ \\
\hline Age at menarche (years) & 14.52 & 1.90 & 14.70 & 1.77 & 0.008 \\
\hline Age at first live birth (years) ${ }^{*}$ & 25.57 & 3.67 & 25.43 & 3.73 & 0.328 \\
\hline Total energy $(\mathrm{kJ} / \mathrm{d})$ & $6050 \cdot 06$ & $1665 \cdot 23$ & 6108.64 & $1652 \cdot 68$ & 0.759 \\
\hline Total energy (kcal/d) & 1446.42 & 398.06 & 1460.58 & 395.03 & 0.759 \\
\hline $\begin{array}{l}\text { Total cruciferous } \\
\text { vegetables }(\mathrm{g} / \mathrm{d}) \dagger\end{array}$ & $115 \cdot 53$ & 71.06 & 130.53 & 68.93 & $<0.001$ \\
\hline Glucosinolate $(\mathrm{mg} / \mathrm{d}) \dagger$ & $89 \cdot 10$ & 63.37 & $100 \cdot 94$ & 59.80 & $<0.001$ \\
\hline \multirow[t]{2}{*}{ Isothiocyanate $(\mu \mathrm{mol} / \mathrm{d}) \dagger$} & $29 \cdot 05$ & $21 \cdot 89$ & $32 \cdot 10$ & $20 \cdot 79$ & $<0.001$ \\
\hline & $n$ & $\%$ & $n$ & $\%$ & \\
\hline Marital status & & & & & 0.277 \\
\hline Married & 1396 & 94.01 & 1401 & 93.03 & \\
\hline $\begin{array}{l}\text { Unmarried/divorced/ } \\
\text { widowed }\end{array}$ & 89 & 5.99 & 105 & 6.97 & \\
\hline Educational level & & & & & 0.004 \\
\hline $\begin{array}{l}\text { Primary school or } \\
\text { below }\end{array}$ & 375 & $25 \cdot 25$ & 388 & $25 \cdot 76$ & \\
\hline Junior high school & 427 & $28 \cdot 75$ & 357 & 23.71 & \\
\hline $\begin{array}{l}\text { Senior high school/ } \\
\text { secondary technical } \\
\text { school }\end{array}$ & 365 & 24.58 & 372 & $24 \cdot 70$ & \\
\hline College or above & 318 & 21.41 & 389 & 25.83 & \\
\hline Occupation & & & & & 0.316 \\
\hline $\begin{array}{l}\text { Administrator/other } \\
\text { white-collar worker }\end{array}$ & 574 & 38.65 & 597 & 39.64 & \\
\hline Blue-collar worker & 380 & 25.59 & 409 & $27 \cdot 16$ & \\
\hline Farmer/other & 531 & $35 \cdot 76$ & 500 & $33 \cdot 20$ & \\
\hline Income (yuan/month) & & & & & 0.015 \\
\hline$<2000$ & 300 & $20 \cdot 20$ & 248 & $16 \cdot 47$ & \\
\hline $2001-5000$ & 440 & 29.63 & 422 & 28.02 & \\
\hline $5001-8000$ & 392 & $26 \cdot 40$ & 442 & $29 \cdot 35$ & \\
\hline$>8001$ & 353 & $23 \cdot 77$ & 394 & $26 \cdot 16$ & \\
\hline Regular smoker & 18 & 1.21 & 8 & 0.53 & 0.045 \\
\hline Passive smoker & 837 & $56 \cdot 36$ & 756 & $50 \cdot 20$ & 0.001 \\
\hline Regular drinker & 189 & $12 \cdot 73$ & 104 & 6.91 & $<0.001$ \\
\hline Occupational activity & & & & & $<0.001$ \\
\hline Non-working & 484 & 32.59 & 402 & $26 \cdot 69$ & \\
\hline Sedentary & 478 & $32 \cdot 19$ & 490 & 32.54 & \\
\hline Standing & 289 & $19 \cdot 46$ & 355 & 23.57 & \\
\hline Manual & 132 & 8.89 & 170 & 11.29 & \\
\hline Heavy manual & 102 & $6 \cdot 87$ & 89 & 5.91 & \\
\hline Menopausal status & & & & & 0.245 \\
\hline Premenopausal & 964 & 64.92 & 1008 & 66.93 & \\
\hline Postmenopausal & 521 & 35.08 & 498 & 33.07 & \\
\hline Parity & & & & & 0.172 \\
\hline 0 & 63 & $4 \cdot 24$ & 72 & 4.78 & \\
\hline $1-2$ & 1085 & 73.06 & 1133 & $75 \cdot 23$ & \\
\hline$\geq 3$ & 337 & 22.69 & 301 & 19.99 & \\
\hline Breast-feeding* & 1259 & 87.50 & 1291 & 87.82 & 0.824 \\
\hline $\begin{array}{l}\text { First-degree relative with } \\
\text { cancer }\end{array}$ & 225 & $15 \cdot 15$ & 118 & $7 \cdot 84$ & $<0.001$ \\
\hline $\begin{array}{l}\text { Ever used an oral } \\
\text { contraceptive }\end{array}$ & 129 & 8.69 & 96 & $6 \cdot 37$ & 0.017 \\
\hline
\end{tabular}

MET, metabolic equivalent task.

* Among women who have had a live birth.

$\dagger$ The consumption was adjusted for total energy intake by the residual method.

quartile was $0.51(95 \%$ CI $0.41,0.63)$ after adjusting for potential confounding factors $\left(P_{\text {trend }}<0 \cdot 001\right)$. Estimated GSL content exposure was also significantly and inversely related to the risk of breast 
Table 2. Risks for breast cancer according to quartiles of cruciferous vegetables, glucosinolates (GSL) and isothiocyanates (ITC) intake (Odds ratios and $95 \%$ confidence intervals)

\begin{tabular}{|c|c|c|c|c|c|c|}
\hline & No. cases/controls & Crude OR & $95 \% \mathrm{Cl}$ & Adjusted OR* & $95 \% \mathrm{Cl}$ & $P_{\text {for trend }}$ \\
\hline Cruciferous vegetables $(\mathrm{g} / \mathrm{d})$ & & & & & & $<0.001$ \\
\hline$<88.80$ & $531 / 376$ & 1.00 & & 1.00 & & \\
\hline $88 \cdot 80-121.06$ & $378 / 377$ & 0.71 & $0.59,0.86$ & 0.74 & $0.61,0.91$ & \\
\hline $121 \cdot 06-164 \cdot 28$ & $311 / 377$ & 0.58 & $0.48,0.71$ & 0.62 & $0.50,0.76$ & \\
\hline$\geq 164.28$ & $265 / 376$ & 0.50 & $0.41,0.61$ & 0.51 & $0.41,0.63$ & \\
\hline GSL (mg/d) & & & & & & $<0.001$ \\
\hline$<59.67$ & $537 / 376$ & 1 & & 1 & & \\
\hline $59 \cdot 67-89 \cdot 78$ & $341 / 376$ & 0.64 & $0.52,0.77$ & 0.67 & $0.55,0.82$ & \\
\hline $89 \cdot 78-130 \cdot 33$ & $323 / 378$ & 0.60 & $0.49,0.73$ & 0.62 & $0.51,0.77$ & \\
\hline$\geq 130.33$ & $284 / 376$ & 0.53 & $0.43,0.65$ & 0.54 & $0.44,0.67$ & \\
\hline ITC $(\mu \mathrm{mol} / \mathrm{d})$ & & & & & & $<0.001$ \\
\hline$<17.59$ & $483 / 376$ & 1 & & 1 & & \\
\hline $17.59-28.00$ & $375 / 377$ & 0.77 & $0.64,0.94$ & 0.83 & $0.67,1.01$ & \\
\hline $28.00-43.01$ & $341 / 377$ & 0.70 & $0.58,0.86$ & 0.74 & $0.60,0.92$ & \\
\hline$\geq 43.01$ & $286 / 376$ & 0.59 & $0.48,0.73$ & 0.62 & $0.50,0.76$ & \\
\hline
\end{tabular}

* OR adjusted for age, education, income, BMI, regular smoking, passive smoking, regular drinking, household and recreational activities, occupational activity, age at menarche, first-degree relative with cancer and ever used an oral contraceptive.

Table 3. Associations between quartiles of cruciferous vegetables, glucosinolate (GSL) and isothiocyanates (ITC) intake and breast cancer risk according to menopausal status

(Odds ratios and $95 \%$ confidence intervals)

\begin{tabular}{|c|c|c|c|c|c|c|c|c|}
\hline & \multirow[b]{2}{*}{ Quartile 1} & \multicolumn{2}{|c|}{ Quartile 2} & \multicolumn{2}{|c|}{ Quartile 3} & \multicolumn{2}{|c|}{ Quartile 4} & \multirow[b]{2}{*}{$P_{\text {for trend }}$} \\
\hline & & OR & $95 \% \mathrm{Cl}$ & OR & $95 \% \mathrm{Cl}$ & OR & $95 \% \mathrm{Cl}$ & \\
\hline \multicolumn{9}{|c|}{ Cruciferous vegetables $(\mathrm{g} / \mathrm{d})$} \\
\hline \multicolumn{9}{|c|}{ Premenopause } \\
\hline No. cases/controls & $361 / 258$ & \multicolumn{2}{|c|}{$246 / 258$} & \multicolumn{2}{|c|}{$195 / 257$} & \multicolumn{2}{|c|}{$162 / 235$} & \\
\hline Crude & 1 & 0.68 & $0.54,0.86$ & 0.54 & $0.42,0.69$ & 0.49 & $0.38,0.64$ & $<0.001$ \\
\hline Adjusted & 1 & 0.70 & $0.55,0.90$ & 0.60 & $0.46,0.77$ & 0.51 & $0.39,0.67$ & $<0.001$ \\
\hline \multicolumn{9}{|l|}{ Postmenopause } \\
\hline No. cases/controls & $170 / 119$ & \multicolumn{2}{|c|}{$132 / 119$} & \multicolumn{2}{|c|}{$116 / 120$} & \multicolumn{2}{|c|}{$103 / 141$} & \\
\hline Crude & 1 & 0.77 & $0.55,1.08$ & 0.67 & $0.47,0.95$ & 0.51 & $0.36,0.72$ & $<0.001$ \\
\hline Adjusted ${ }^{\star}$ & 1 & 0.81 & $0.57,1.16$ & 0.63 & $0.44,0.91$ & 0.50 & $0.34,0.72$ & $<0.001$ \\
\hline \multicolumn{9}{|l|}{$\mathrm{GSL}(\mathrm{mg} / \mathrm{d})$} \\
\hline \multicolumn{9}{|l|}{ Premenopause } \\
\hline No. cases/controls & $362 / 263$ & \multicolumn{2}{|c|}{$224 / 263$} & \multicolumn{2}{|c|}{$207 / 241$} & \multicolumn{2}{|c|}{$171 / 241$} & \\
\hline Crude & 1 & 0.62 & $0.49,0.79$ & 0.62 & $0.49,0.80$ & 0.52 & $0.40,0.66$ & $<0.001$ \\
\hline Adjusted $^{\star}$ & 1 & 0.65 & $0.51,0.84$ & 0.66 & $0.51,0.86$ & 0.55 & $0.42,0.72$ & $<0.001$ \\
\hline \multicolumn{9}{|l|}{ Postmenopause } \\
\hline No. cases/controls & $175 / 113$ & \multicolumn{2}{|c|}{$117 / 113$} & \multicolumn{2}{|c|}{$116 / 137$} & \multicolumn{2}{|c|}{$113 / 135$} & \\
\hline Crude & 1 & 0.67 & $0.47,0.95$ & 0.55 & $0.39,0.77$ & 0.54 & $0.38,0.76$ & $<0.001$ \\
\hline Adjusted* & 1 & 0.68 & $0.47,0.98$ & 0.52 & $0.36,0.74$ & 0.52 & $0.36,0.75$ & $<0.001$ \\
\hline \multicolumn{9}{|l|}{ ITC $(\mu \mathrm{mol} / \mathrm{d})$} \\
\hline \multicolumn{9}{|l|}{ Premenopause } \\
\hline No. cases/controls & $316 / 280$ & \multicolumn{2}{|c|}{$253 / 250$} & \multicolumn{2}{|c|}{$220 / 236$} & \multicolumn{2}{|c|}{$175 / 242$} & \\
\hline Crude & 1 & 0.90 & $0.71,1.14$ & 0.83 & $0.65,1.05$ & 0.64 & $0.50,0.83$ & 0.001 \\
\hline Adjusted* & 1 & 0.96 & $0.75,1.23$ & 0.89 & $0.69,1.15$ & 0.70 & $0.53,0.91$ & 0.010 \\
\hline \multicolumn{9}{|l|}{ Postmenopause } \\
\hline No. cases/controls & $167 / 96$ & \multicolumn{2}{|c|}{$122 / 127$} & & 1/141 & & 1/134 & \\
\hline Crude & 1 & 0.55 & $0.39,0.79$ & 0.49 & $0.35,0.7$ & 0.48 & $0.33,0.68$ & $<0.001$ \\
\hline Adjusted ${ }^{\star}$ & & 0.55 & $0.37,0.80$ & 0.46 & $0.32,0.67$ & 0.45 & $0.31,0.66$ & $<0.001$ \\
\hline
\end{tabular}

* OR adjusted for age, education, income, BMI, regular smoking passive smoker, regular drinking, household and recreational activities, occupational activity, age at menarche, first-degree relative with cancer and ever used an oral contraceptive.

cancer (highest $v$. lowest quartile: $\mathrm{OR}=0.54 ; 95 \%$ CI 0.44, 0.67, $\left.P_{\text {trend }}<0 \cdot 001\right)$. Similarly, women with high exposure of ITC had significantly lower risk of breast cancer (highest $v$. lowest quartile: adjusted $\left.\mathrm{OR}=0.62 ; 95 \% \mathrm{CI} 0.50,0.76, P_{\text {trend }}<0.001\right)$.

In all, there were 1972 premenopausal women (964 cases and 1008 controls) and 1019 postmenopausal women (521 cases and 498 controls). Stratified analysis by menopausal status (Table 3) showed a notably inverse association in both premenopausal and postmenopausal women with high intakes of cruciferous vegetables. Compared with the lowest quartile, the adjusted OR in the highest quartile were 0.51 (95\% CI 0.41 , $\left.0.67, P_{\text {trend }}<0.001\right)$ among premenopausal women and 0.50 (95\% CI $\left.0.34,0.72, P_{\text {trend }}<0.001\right)$ among postmenopausal women. Analogously, the inverse associations between intakes of GSL, ITC and breast cancer risk were also observed in both premenopausal and postmenopausal women. 
Table 4. Associations between quartiles of cruciferous vegetables, glucosinolate (GSL) and isothiocyanates (ITC) intake and breast cancer risk according to sex hormone receptor status

(Odds ratios and $95 \%$ confidence intervals)

\begin{tabular}{|c|c|c|c|c|c|c|c|c|}
\hline & \multirow[b]{2}{*}{ Quartile 1} & \multicolumn{2}{|c|}{ Quartile 2} & \multicolumn{2}{|c|}{ Quartile 3} & \multicolumn{2}{|c|}{ Quartile 4} & \multirow[b]{2}{*}{$P_{\text {for trenc }}$} \\
\hline & & OR & $95 \% \mathrm{Cl}$ & OR & $95 \% \mathrm{Cl}$ & OR & $95 \% \mathrm{Cl}$ & \\
\hline \multicolumn{9}{|c|}{ Cruciferous vegetables $(\mathrm{g} / \mathrm{d})$} \\
\hline \multicolumn{9}{|c|}{$E R+P R+$} \\
\hline No. cases/controls & $304 / 377$ & \multicolumn{2}{|c|}{$210 / 377$} & \multicolumn{2}{|c|}{$176 / 377$} & \multicolumn{2}{|c|}{$141 / 376$} & \\
\hline Crude & 1 & 0.69 & $0.55,0.86$ & 0.58 & $0.46,0.73$ & 0.46 & $0.36,0.59$ & $<0.001$ \\
\hline Adjusted* & 1 & 0.71 & $0.56,0.90$ & 0.61 & $0.48,0.78$ & 0.48 & $0.39,0.62$ & $<0.001$ \\
\hline \multicolumn{9}{|l|}{$\mathrm{ER}+\mathrm{PR}-$} \\
\hline No. cases/controls & 28/377 & \multicolumn{2}{|c|}{$19 / 377$} & \multicolumn{2}{|c|}{$15 / 377$} & \multicolumn{2}{|c|}{$16 / 376$} & \\
\hline Crude & 1 & 0.68 & $0.37,1.23$ & 0.53 & $0.28,1.02$ & 0.57 & $0.30,1.07$ & 0.050 \\
\hline Adjusted* & 1 & 0.69 & $0.37,1.27$ & 0.57 & $0.30,1.12$ & 0.59 & $0.31,1.14$ & 0.082 \\
\hline \multicolumn{9}{|l|}{$\mathrm{ER}-\mathrm{PR}+$} \\
\hline No. cases/controls & 29/377 & \multicolumn{2}{|c|}{$20 / 377$} & \multicolumn{2}{|c|}{$14 / 377$} & \multicolumn{2}{|c|}{$17 / 376$} & \\
\hline Crude & 1 & 0.69 & $0.38,1.24$ & 0.48 & $0.25,0.93$ & 0.57 & $0.32,1.09$ & 0.042 \\
\hline Adjusted* & 1 & 0.68 & $0.37,1.23$ & 0.53 & $0.28,1.02$ & 0.57 & $0.30,1.07$ & 0.224 \\
\hline ER-PR- & & & & & & & & \\
\hline No. cases/controls & $80 / 377$ & & 377 & & 377 & & 376 & \\
\hline Crude & 1 & 0.74 & $0.51,1.06$ & 0.45 & $0.30,0.68$ & 0.48 & $0.32,0.72$ & $<0.001$ \\
\hline Adjusted* & 1 & 0.73 & $0.50,1.06$ & 0.49 & $0.32,0.76$ & 0.50 & $0.33,0.78$ & $<0.001$ \\
\hline $\mathrm{GSL}(\mathrm{mg} / \mathrm{d})$ & & & & & & & & \\
\hline $\mathrm{ER}+\mathrm{PR}+$ & & & & & & & & \\
\hline No. cases/controls & $307 / 376$ & & /376 & & /378 & & 376 & \\
\hline Crude & 1 & 0.60 & $0.47,0.75$ & 0.58 & $0.46,0.73$ & 0.53 & $0.42,0.67$ & $<0.001$ \\
\hline Adjusted* & 1 & 0.63 & $0.50,0.80$ & 0.60 & $0.47,0.76$ & 0.55 & $0.43,0.71$ & $<0.001$ \\
\hline$E R+P R-$ & & & & & & & & \\
\hline No. cases/controls & 29/377 & & 377 & & 377 & & & \\
\hline Crude & 1 & 0.52 & $0.23,0.98$ & 0.69 & $0.38,1.23$ & 0.48 & $0.25,0.93$ & 0.049 \\
\hline Adjusted* & 1 & 0.53 & $0.27,1.01$ & 0.72 & $0.39,1.32$ & 0.51 & $0.26,1.00$ & 0.612 \\
\hline ER-PR+ & & & & & & & & \\
\hline No. cases/controls & $28 / 377$ & & 377 & & 377 & & 376 & \\
\hline Crude & 1 & 0.71 & $0.40,1.29$ & 0.53 & $0.28,1.01$ & 0.61 & $0.33,1.13$ & 0.065 \\
\hline Adjusted* & 1 & 0.76 & $0.41,1.40$ & 0.60 & $0.31,1.18$ & 0.78 & $0.41,1.50$ & 0.316 \\
\hline ER-PR- & & & & & & & & \\
\hline No. cases/controls & $75 / 376$ & & 376 & & 378 & & 376 & \\
\hline Crude & 1 & 0.87 & $0.60,1.24$ & 0.52 & $0.34,0.78$ & 0.45 & $0.30,0.70$ & $<0.001$ \\
\hline Adjusted* & 1 & 0.84 & $0.58,1.23$ & 0.53 & $0.35,0.82$ & 0.48 & $0.30,0.75$ & $<0.001$ \\
\hline ITC $(\mu \mathrm{mol} / \mathrm{d})$ & & & & & & & & \\
\hline $\mathrm{ER}+\mathrm{PR}+$ & & & & & & & & \\
\hline No. cases/controls & $275 / 376$ & & $/ 377$ & & $/ 377$ & & $/ 376$ & \\
\hline Crude & 1 & 0.75 & $0.59,0.94$ & 0.67 & $0.53,0.84$ & 0.60 & $0.48,0.77$ & $<0.001$ \\
\hline Adjusted* & 1 & 0.80 & $0.63,1.01$ & 0.71 & $0.60,0.91$ & 0.64 & $0.50,0.82$ & $<0.001$ \\
\hline $\mathrm{ER}+\mathrm{PR}-$ & & & & & & & & \\
\hline No. cases/controls & $29 / 376$ & & 377 & & 377 & & 376 & \\
\hline Crude & 1 & 0.45 & $0.23,0.87$ & 0.65 & $0.36,1.19$ & 0.59 & $0.32,1.09$ & 0.141 \\
\hline Adjusted* & 1 & 0.50 & $0.25,0.98$ & 0.71 & $0.38,1.32$ & 0.65 & $0.34,1.24$ & 0.253 \\
\hline $\mathrm{ER}-\mathrm{PR}+$ & & & & & & & & \\
\hline No. cases/controls & $23 / 377$ & & 377 & & 377 & & 376 & \\
\hline Crude & 1 & $1 \cdot 12$ & $0.69,2 \cdot 15$ & 0.52 & $0.26,1.06$ & 0.74 & $0.39,1.41$ & 0.099 \\
\hline Adjusted* & 1 & 1.29 & $0.71,2.33$ & 0.61 & $0.29,1.27$ & 0.94 & $0.48,1.85$ & 0.412 \\
\hline ER-PR- & & & & & & & & \\
\hline No. cases/controls & $63 / 376$ & & 377 & & 377 & & 376 & \\
\hline Crude & 1 & 1.09 & $0.75,1.58$ & 0.73 & $0.49,1.09$ & 0.56 & $0.36,0.86$ & 0.002 \\
\hline Adjusted* & 1 & 1.09 & $0.74,1.60$ & 0.76 & $0.49,1.16$ & $0 \cdots 61$ & $0.38,0.96$ & 0.013 \\
\hline
\end{tabular}

ER+, oestrogen receptor-positive; ER-, oestrogen receptor negative; PR+, progesterone receptor positive; PR-, progesterone receptor negative.

* OR adjusted for age, education, income, BMI, regular smoking, passive smoking, regular drinking, household and recreational activities, occupational activity, age at menarche, first-degree relative with cancer and ever used an oral contraceptive.

When cases were grouped on the basis of sex hormone receptor status, 831 cases were in $\mathrm{ER}+\mathrm{PR}+$ status, seventy-eight cases in ER+ PR- status, eighty cases in ER- PR+ status and 213 cases in ER- PR- status. Among cases with ER+ PR+ and ER- PR- status, intakes of cruciferous vegetables, GSL and ITC were inversely associated with breast cancer risk. For the ER+ PR- and ER- PR+ subtype, a non-significant inverse association was found between cruciferous vegetables, GSL, ITC and breast cancer risk (Table 4).

\section{Discussion}

Our case-control study among Chinese women revealed an inverse association between cruciferous vegetable intake and 
breast cancer risk. Estimated GSL and ITC content exposure was also inversely associated with the risk of breast cancer.

In the present study, ten types of cruciferous vegetables including choi sum, kaichoi, kailan, broccoli, pakchoi, bokchoi, cabbage, mini Chinese cabbage, cauliflower and radish common in Guangdong province were included. The inverse association between cruciferous vegetable consumption and breast cancer risk observed in the present study was in agreement with the meta-analysis published in $2013^{(10)}$. However, no statistically significant association between cruciferous vegetable intake and breast cancer risk was found in most studies conducted in Western populations ${ }^{(28-35)}$. Some possible explanations might account for the different results. First, the level of cruciferous vegetable consumption differed among different populations. The energy-adjusted median intake of cruciferous vegetables in our population was $130 \mathrm{~g} / \mathrm{d}$, which was $>3 \mathrm{~g} / \mathrm{d}$ in Japanese women ${ }^{(30)}, 11.3 \mathrm{~g} / \mathrm{d}$ in the Spanish cohort of the European Prospective Investigation into Cancer and Nutrition (EPIC) ${ }^{(36)}$ and $31 \mathrm{~g} / \mathrm{d}$ in women from the Nurses' Health Study ${ }^{(37)}$. The lack of association between consumption of cruciferous vegetables and breast cancer risk in Western populations might be owing to their much lower level of consumption. Second, owing to the difference of dietary patterns between China and Western countries ${ }^{(38)}$, the types of cruciferous vegetables consumed varied in different countries. For example, broccoli, cauliflower, cabbage, collard green and Brussels sprout are commonly consumed in Western countries, whereas choi sum, kaichoi, kailan, pakchoi and bokchoi are mostly consumed in Asian diets ${ }^{(39)}$

To our knowledge, no epidemiological study has yet been published to examine the association between GSL intake and breast cancer risk. Only the EPIC cohort ${ }^{(40)}$, which is a multicentre prospective cohort study comprising 11405 men, assessed the relationship between dietary GSL intake and prostate cancer risk. A significant inverse association was found between GSL intake and prostate cancer risk, with an adjusted hazard ratios of 0.68 (95\% CI $0.48,0.97$ ) comparing the highest with the lowest quartile. In this study, a notable $46 \%$ reduction of breast cancer risk was observed among women with a high intake of the GSL obtained from the cruciferous vegetables commonly consumed in China.

As a vital bioactive breakdown component of GSL, the association between ITC intake and breast cancer has been investigated in only a few epidemiological studies. Consistent with the result of the Shanghai Breast Cancer Study ${ }^{(41)}$, our result suggested a $38 \%$ reduction in breast cancer risk with ITC intake. Besides, two population-based case-control studies found an inverse association between ITC and lung cancer risk in both African-American and Caucasian women from America $^{(16)}$, and Chinese women from Singapore ${ }^{(17)}$. However, no significant association between ITC intake and oral cancer risk was found in a hospital-based case-control study consisting of 115 oral cancer cases and 116 controls conducted in Malaysia $^{(42)}$.

Cruciferous vegetables contain numerous nutrients and phytochemicals with cancer chemo-preventive properties, including carotenoids, folate, fibre and chlorophyll ${ }^{(12)}$. Furthermore, what makes cruciferous vegetables unique are rich sources of GSL. Nearly 120 GSL with unique breakdown products have been identified in cruciferous vegetables ${ }^{(43)}$. Owing to the chemical and thermal stability of most GSL, the hydrolysis of GSL relies on plant myrosinase, which exists physically separated from GSL in plant cells. When intact cruciferous vegetables are chopped or chewed, GSL come in contact with plant myrosinase, resulting in the formation of ITC at a neutral $\mathrm{pH}$ of $6-7^{(44)}$. ITC exert their chemoprotective effect by two major mechanisms. One mechanism is inhibiting the biotransformation of chemical carcinogens by specific CYP enzymes to active forms that are capable of binding DNA to induce mutations. The other mechanism is that ITC potentially induced phase II enzyme activity, primarily GST, by increasing the transcription of genes that contain an antioxidant response element ${ }^{(45)}$. ITC are water-soluble compounds that may be leached into cooking water, and thus we estimated dietary ITC intake by using previously published ITC concentrations for cruciferous vegetables cooked in boiling water ${ }^{(25)}$.

Stratified analysis by menopausal status found no disparity between premenopausal and postmenopausal women. Our findings are also consistent with those of an intervention study ${ }^{(46)}$, which showed that the addition of $500 \mathrm{~g} / \mathrm{d}$ brassica to the daily diet of thirty-four healthy postmenopausal women may shift oestrogen metabolism, as measured by the increased values of the biomarker ratio 2-hydroxyoestrone:16 $\alpha$-hydroxyoestrone in urine. Compared with 2-hydroxyoestrone, 16 $\alpha$ hydroxyoestrone is relatively more oestrogenic and genotoxic. Therefore, this change in oestrogen metabolism may be a mechanism to protect against breast cancer ${ }^{(47)}$. Similar results were found in another small sample intervention study ${ }^{(48)}$, which reported that eleven of the thirteen premenopausal women showed strongly positive increases in their 2-hydroxyoestrone:16 $\alpha$-hydroxyoestrone ratios in urine after using the supplements of dried cruciferous powder for $90 \mathrm{~d}$. However, a recent meta-analysis of eleven case-control studies and two cohort studies examining the associations between cruciferous vegetables and breast cancer risk ${ }^{(10)}$ showed that the protective effect of cruciferous vegetables against breast cancer risk was present only in postmenopausal women. As there were only five studies in the premenopausal group and the heterogeneity was high $(P=0.004)$, the results are still inconclusive. More studies are needed to clarify this association of cruciferous vegetables and breast cancer risk defined by menopausal status.

In this study, higher consumption of cruciferous vegetables, GSL and ITC was found to be inversely associated with breast cancer risk in women with both hormone-receptor-positive or hormone-receptor-negative status. Consistent with our results, a cohort study conducted in Japan ${ }^{(30)}$ reported that cruciferous vegetable intake was marginally inversely associated with ER+ and PR+ tumours. The adjusted relative risk was 0.64 (95\% CI $0 \cdot 41,1 \cdot 00)$, comparing the highest with the lowest quartile. The Pooling Project of Prospective Studies of Diet and Cancer, comprising twenty cohort studies ${ }^{(49)}$, indicated that total vegetable consumption was significantly and inversely associated with the risk of ER- breast cancer (highest $v$. lowest quintile: adjusted $\mathrm{OR}=0.82 ; 95 \%$ CI $0 \cdot 74,0 \cdot 90)$. Similar result was found in EPIC cohort study ${ }^{(50)}$ and the Nurses' Health Study ${ }^{(51)}$. The rational biological mechanism is that ITC can affect hormonal 
level through inhibiting the transcription of oestrogen-simulated target genes such as ER and PR, thus preventing the development of ER+ PR+ tumours ${ }^{(52)}$. The mechanism for lowering ER- tumours might be hormonally independent. It was reported that ER- tumours are more likely to be less dependent on oestrogen levels than ER+ tumours ${ }^{(52)}$. Studies showed that NF$\kappa \mathrm{B}$, epidermal growth factor receptor and cyclin $\mathrm{E}$ were overexpressed in ER- breast tumours, leading to the development of breast cancer. Bioactive components contained in cruciferous vegetables might reduce the levels of these factors ${ }^{(53)}$ and lower the risk of ER- breast cancer. Our results showed a non-significant inverse association among the ER+ PR- and ER- PR+subtypes. However, as the cases in the subgroups are relatively small, further studies are needed to clarify this issue.

The strengths of the present study include the relatively large sample size, and the satisfactory reproducibility and reasonable validity of the FFQ. In addition, this is the first study to evaluate the association between cruciferous vegetable intakes and breast cancer risk according to GSL and ITC contents. Our attempt to measure GSL and ITC intake based on various cruciferous vegetables commonly consumed locally further strengthen the study findings.

The study has a few limitations. First, the results could be affected by recall bias and selection bias, which are inevitable in hospital-based case-control studies. To minimise selection bias, control subjects were recruited to exclude any disease potentially related either to breast cancer or dietary changes. However, the time-concordant period of hospitalisation, identical catchment areas of all subjects and the relatively high response rate helped to reduce the selection bias. Moreover, to minimise recall bias, cases were interviewed as soon as the diagnosis was made and as far as possible before their surgery. In addition, food photographs were provided to assist participants with quantification of dietary intake. Second, residual confounding remained even though various dietary and non-dietary confounders were adjusted in the multivariable models. Third, this study did not measure urinary ITC, which have been shown to be useful biomarkers for recent cruciferous vegetable intake $^{(54,55)}$. Further studies are needed to assess the association between cruciferous vegetable intake and breast cancer risk by measuring urinary ITC.

In conclusion, this study indicated that higher cruciferous vegetables, GSL and ITC intakes were inversely associated with breast cancer risk among Chinese women. The findings suggested the protective role of cruciferous vegetables, rich in GSL and ITC, against breast cancer.

\section{Acknowledgements}

The authors gratefully acknowledge the contribution of the study participants, without whom the study would not have been possible.

This study was supported by Danone Nutrition Research and Education Foundation (no: DIC2017-05). The funders had no role in the design, analysis or writing of this article.

The authors' responsibilities were as follows: N.-Q. Z. conducted the data collection, analysed the data and writing of this paper. S. C. H. provided significant advice regarding the analyses and interpretation of the data. W.-Q. H., H. L. and J. H., participated in the data collection and data entry. X.-F. M. and F.-Y. L. were responsible for connecting and coordinating the field work. C.-X. Z. constructed the project design, and supervised and contributed to manuscript writing.

The authors declare that there are no conflicts of interest.

\section{References}

1. Torre LA, Bray F \& Siegel RL (2015) Global cancer statistics, 2012. CA Cancer J Clin 65, 87-108.

2. Torre LA, Siegel RL \& Ward EM (2016) Global cancer incidence and mortality rates and trends - an update. Cancer Epidemiol Biomarkers Prev 25, 16-27.

3. Wu Q, Yang Y \& Wang J (2013) Cruciferous vegetable consumption and gastric cancer risk: A meta-analysis of epidemiological studies. Cancer Sci 104, 1067-1073.

4. Li LY, Luo Y \& Lu MD (2015) Cruciferous vegetable consumption and the risk of pancreatic cancer: a meta-analysis. World J Surg Oncol 13, 44

5. Tse G \& Eslick GD (2014) Cruciferous vegetables and risk of colorectal neoplasms: a systematic review and meta-analysis. Nutr Cancer 66, 128-139.

6. Liu B, Mao Q \& Wang X (2013) Cruciferous vegetables consumption and risk of renal cell carcinoma: a meta-analysis. Nutr Cancer 65, 668-676.

7. Wu QJ, Xie L \& Zheng W (2013) Cruciferous vegetables consumption and the risk of female lung cancer: a prospective study and a meta-analysis. Ann Oncol 24, 1918-1924.

8. Liu B, Mao Q \& Cao M (2012) Cruciferous vegetables intake and risk of prostate cancer: a meta-analysis. Int J Urol 19, 134-141.

9. Wu QJ, Yang Y \& Vogtmann E (2013) Cruciferous vegetables intake and the risk of colorectal cancer: a meta-analysis of observational studies. Ann Oncol 24, 1079-1087.

10. Liu X \& Lv K (2013) Cruciferous vegetables intake is inversely associated with risk of breast cancer: a meta-analysis. Breast 22, 309-313.

11. Liu B, Mao Q \& Lin Y (2013) The association of cruciferous vegetables intake and risk of bladder cancer: a meta-analysis. World J Urol 31, 127-133.

12. Abdull Razis AF \& Noor NM (2013) Cruciferous vegetables: dietary phytochemicals for cancer prevention. Asian Pac J Cancer Prev 14, 1565-1570.

13. Zhang Y \& Talalay P (1994) Anticarcinogenic activities of organic isothiocyanates: chemistry and mechanisms. Cancer Res 54, 1976s-1981s.

14. Singh SV \& Singh K (2012) Cancer chemoprevention with dietary isothiocyanates mature for clinical translational research. Carcinogenesis 33, 1833-1842.

15. Steinbrecher A, Rohrmann S \& Timofeeva M (2010) Dietary glucosinolate intake, polymorphisms in selected biotransformation enzymes, and risk of prostate cancer. Cancer Epidemiol Biomarkers Prev 19, 135-143.

16. Carpenter CL, Yu MC \& London SJ (2009) Dietary isothiocyanates, glutathione S-transferase M1 (GSTM1), and lung cancer risk in African Americans and Caucasians from Los Angeles County, California. Nutr Cancer 61, 492-499.

17. Zhao B, Seow A \& Lee E (2001) Dietary isothiocyanates, glutathione S-transferase-M1,-T1 polymorphisms and lung cancer risk among Chinese women in Singapore. Cancer Epidemiol Biomarkers Prev 10, 1063-1067.

18. Zhang C, Ho SC \& Lin F (2010) Soy product and isoflavone intake and breast cancer risk defined by hormone receptor status. Cancer Sci 101, 501-507. 
19. Zhang C, Ho SC \& Chen Y (2009) Greater vegetable and fruit intake is associated with a lower risk of breast cancer among Chinese women. Int J Cancer 125, 181-188.

20. Ainsworth BE, Haskell WL \& Whitt MC (2000) Compendium of physical activities: an update of activity codes and MET intensities. Med Sci Sports Exerc 32, S498-S504.

21. Ainsworth BE, Haskell WL \& Herrmann SD (2011) 2011 Compendium of Physical Activities: a second update of codes and MET values. Med Sci Sports Exerc 43, 1575-1581.

22. Zhang CX \& Ho SC (2009) Validity and reproducibility of a food frequency questionnaire among Chinese women in Guangdong province. Asia Pac J Clin Nutr 18, 240-250.

23. Yue-xin Y, Guangya W \& Xingchang P (2002) China Food Composition 2002. Beijing: Institute of Nutrition and Food Safety, Peking University Medical Press.

24. McNaughton SA \& Marks GC (2003) Development of a food composition database for the estimation of dietary intakes of glucosinolates, the biologically active constituents of cruciferous vegetables. Br J Nutr 90, 687.

25. Jiao D, Yu MC \& Hankin JH (1998) Total isothiocyanate contents in cooked vegetables frequently consumed in Singapore. J Agric Food Chem 46, 1055-1058.

26. Willett WC, Howe GR \& Kushi LH (1997) Adjustment for total energy intake in epidemiologic studies. Am J Clin Nutr 65 , 1220S-1228S, 1229S-1231S.

27. Collaborative Group on Hormonal Factors in Breast Cancer (2012) Menarche, menopause, and breast cancer risk: individual participant meta-analysis, including 118964 women with breast cancer from 117 epidemiological studies. Lancet Oncol 13, 1141-1151

28. Terry P, Wolk A \& Persson I (2001) Brassica vegetables and breast cancer risk. JAMA 285, 2975-2977.

29. Shannon J, Cook LS \& Stanford JL (2003) Dietary intake and risk of postmenopausal breast cancer (United States). Cancer Causes Control 14, 19-27.

30. Suzuki R, Iwasaki M \& Hara A (2013) Fruit and vegetable intake and breast cancer risk defined by estrogen and progesterone receptor status: the Japan Public Health Center-based Prospective Study. Cancer Causes Control 24, 2117-2128.

31. Boggs DA, Palmer JR \& Wise LA (2010) Fruit and vegetable intake in relation to risk of breast cancer in the Black Women's Health Study. Am J Epidemiol 172, 1268-1279.

32. Potischman N, Swanson CA \& Coates RJ (1999) Intake of food groups and associated micronutrients in relation to risk of early-stage breast cancer. Int J Cancer 82, 315-321.

33. Franceschi S, Parpinel M \& La Vecchia C (1998) Role of different types of vegetables and fruit in the prevention of cancer of the colon, rectum, and breast. Epidemiology 9, 338-341.

34. Byers $\mathrm{T} \&$ \& Graham S (1984) The epidemiology of diet and cancer. Adv Cancer Res 41, 1-69.

35. Rosenblatt KA, Thomas DB \& Jimenez LM (1999) The relationship between diet and breast cancer in men (United States). Cancer Causes Control 10, 107-113.

36. Agudo A, Ibanez R \& Amiano P (2008) Consumption of cruciferous vegetables and glucosinolates in a Spanish adult population. Eur J Clin Nutr 62, 324-331.

37. Kang JH, Ascherio A \& Grodstein F (2005) Fruit and vegetable consumption and cognitive decline in aging women. Ann Neurol 57, 713-720.
38. Albuquerque RC, Baltar VT \& Marchioni DM (2014) Breast cancer and dietary patterns: a systematic review. Nutr Rev $\mathbf{7 2}$, $1-17$.

39. Tang L, Paonessa JD \& Zhang Y (2013) Total isothiocyanate yield from raw cruciferous vegetables commonly consumed in the United States. J Funct Foods 5, 1996-2001.

40. Steinbrecher A, Nimptsch K \& Husing A (2009) Dietary glucosinolate intake and risk of prostate cancer in the EPICHeidelberg cohort study. Int J Cancer 125, 2179-2186.

41. Lee SA, Fowke JH \& Lu W (2008) Cruciferous vegetables, the GSTP1 Ile105Val genetic polymorphism, and breast cancer risk. Am J Clin Nutr 87, 753-760.

42. Karen-Ng LP, Marhazlinda J \& Rahman ZAA (2011) Combined effects of isothiocyanate intake, glutathione S-transferase polymorphisms and risk habits for age of oral squamous cell carcinoma development. Asian Pac J Cancer Prev 12, 1161-1166.

43. Conzatti A, Froes FC \& Schweigert PI (2014) Clinical and molecular evidence of the consumption of broccoli, glucoraphanin and sulforaphane in humans. Nutr Hosp 31, 559-569.

44. Shapiro TA, Fahey JW \& Wade KL (2001) Chemoprotective glucosinolates and isothiocyanates of broccoli sprouts: metabolism and excretion in humans. Cancer Epidemiol Biomarkers Prev 10, 501-508.

45. Kensler TW, Egner PA \& Wang JB (2004) Chemoprevention of hepatocellular carcinoma in aflatoxin endemic areas. Gastroenterology 127, S310-S318.

46. Fowke JH, Longcope C \& Hebert JR (2000) Brassica vegetable consumption shifts estrogen metabolism in healthy postmenopausal women. Cancer Epidemiol Biomarkers Prev 9, 773-779.

47. Ziegler RG, Fuhrman BJ \& Moore SC (2015) Epidemiologic studies of estrogen metabolism and breast cancer. Steroids 99 , $67-75$.

48. Morrison J, Mutell D \& Pollock TA (2009) Effects of dried cruciferous powder on raising $2 / 16$ hydroxyestrogen ratios in premenopausal women. Altern Ther Health Med 15, $52-53$.

49. Jung S, Spiegelman D \& Baglietto L (2013) Fruit and vegetable intake and risk of breast cancer by hormone receptor status. J Natl Cancer Inst 105, 219-236.

50. Emaus MJ, Peeters PH \& Bakker MF (2016) Vegetable and fruit consumption and the risk of hormone receptor-defined breast cancer in the EPIC cohort. Am J Clin Nutr 103, 168-177.

51. Fung TT, Chiuve SE \& Willett WC (2013) Intake of specific fruits and vegetables in relation to risk of estrogen receptornegative breast cancer among postmenopausal women. Breast Cancer Res Treat 138, 925-930.

52. Yip CH \& Rhodes A (2014) Estrogen and progesterone receptors in breast cancer. Future Oncol 10, 2293-2301.

53. Johnson IT (2007) Phytochemicals and cancer. Proc Nutr Soc 66, 207-215.

54. Fowke JH, Chung FL \& Jin F (2003) Urinary isothiocyanate levels, brassica, and human breast cancer. Cancer Res $\mathbf{6 3}$, 3980-3986.

55. Fowke JH, Fahey JW \& Stephenson KK (2001) Using isothiocyanate excretion as a biological marker of Brassica vegetable consumption in epidemiological studies: evaluating the sources of variability. Public Health Nutr $\mathbf{4}, 837$. 